\title{
CLASSIFICATION OF HUMAN ACTIONS INTO DYNAMICS BASED PRIMITIVES WITH APPLICATION TO DRAWING TASKS
}

\author{
D. Del Vecchio*, R. M. Murray*, P. Perona* \\ * Division of Engineering and Applied Science \\ California Institute of Technology \\ Pasadena, CA 91125, ddomitilla@cds.caltech.edu
}

Keywords: Classification, parameter estimation, data analysis, computer experiments

\begin{abstract}
We develop the study of primitives of human motion, which we refer to as movemes. The idea is to understand human motion by decomposing it into a sequence of elementary building blocks that belong to a known alphabet of dynamical systems. How can we construct an alphabet of movemes from human data? In this paper we address this issue by introducing the notion of well-posednes. Using examples from human drawing data, we show that the well-posedness notion can be applied in practice so to establish if sets of actions, viewed as signals in time, can define movemes.
\end{abstract}

\section{Introduction}

Building systems that can detect and recognize human actions and activities is an important goal of modern engineering. Applications range from human-machine interfaces to security to entertainment.

A fundamental problem in detecting and recognizing human action is one of representation. As explained in [4], our point of view is that human activity should be decomposed into building blocks which belong to an "alphabet" of elementary actions. We refer to these primitives of motion as movemes. We thus aim to build an alphabet of movemes which one can compose to represent and describe human motion similar to the way phonemes are used in speech. The word "moveme" intended as primitive of motion was invented by [2]. They studied periodic or stereotypical motions such as walking or running where the motion is always the same and therefore their movemes, like the phonemes, were repeatable segments of trajectory. [5] studied motions that were parametrized by an initial condition and a target. They proposed that movemes ought to be parametrized by goal and style parameters. Their moveme models are phenomenological and non-causal.

What is the alphabet of movemes? Which are the dynamical models that we should use to represent them? Where do movemes come from in practice? When human actions can define movemes according to a dynamical model class $M$ ? To answer these questions we use system identification tools [6, 7], we recall the formal definition of moveme already given in [4], and we introduce the classification problem as a standard problem of pattern recognition [1, 8]. In [4] and [3] some classification results have been presented as instrumental for the segmentation problem. However the assumption that the actions considered were defining movemes was tacitly made. In this paper we show with examples from real data that such an assumption may not hold in practice and we explain the reason. We thus propose a way to establish when real data (seen as signals in time) can allow the definition of a set of movemes. To this regard we introduce the definition of well-posed sets of signals, and we show how such a definition can be checked in practice. Experiments on drawing data are reported.

\section{Dynamical Definition of Moveme}

We recall in this section a relaxed version of the definition of moveme already presented in [4], we introduce the model class, and we set the classification problem.

\subsection{Definitions and properties}

Let $M(\Theta)$ denote a linear time invariant (LTI) system class parameterized by $\Theta \in E, E$ a linear space, and let $\mathcal{U}$ denote a class of inputs. Let $y(t)=Y\left(\left.M(\Theta)\right|_{u, x_{0}}\right)(t)$, for $t \geq t_{0}$, denote the output of $M(\Theta)$ once parameter $\Theta \in E$, input $u \in$ $\mathcal{U}$, and initial conditions $x_{0}$ have been chosen. Let $\theta \in E^{\prime} \subseteq E$ be a parameter lying in a subspace of $E$, and define a map $\Upsilon$ : $E \rightarrow E^{\prime}$. We write $\theta=\Upsilon(\Theta)$ to represent the transformation from $\Theta \in E$ to the reduced set of parameters $\theta \in E^{\prime}$.

Definition 2.1. Let $M^{1}=\left\{M(\Theta) \mid \theta \in \mathcal{C}^{1}\right\}$ and $M^{2}=$ $\left\{M(\Theta) \mid \theta \in \mathcal{C}^{2}\right\}$ denote two subsets in $M$ with $\mathcal{C}^{j} \subset E^{\prime}$ for $j=1,2 . M^{1}$ and $M^{2}$ are said to be dynamically independent if

(i) the class of systems $M$ and the class of inputs $\mathcal{U}$ are such that

$$
Y\left(\left.M\left(\Theta_{1}\right)\right|_{u_{1}, x_{0}}\right)(t)=Y\left(\left.M\left(\Theta_{2}\right)\right|_{u_{2}, x_{0}}\right)(t),
$$

for all $t \geq t_{0}$, if and only if $\left(\Theta_{1}, u_{1}\right)=\left(\Theta_{2}, u_{2}\right)$ for $u_{1} \in \mathcal{U}$ and $u_{2} \in \mathcal{U}$;

(ii) the sets $\mathcal{C}^{1}$ and $\mathcal{C}^{2}$ are non empty, bounded, and have trivial intersection, i.e. $\mathcal{C}^{1} \cap \mathcal{C}^{2}=\{\emptyset\}$. 
Each of the elements of a set $\mathcal{M}=\left\{M^{1}, \ldots, M^{l}\right\}$ of mutually dynamically independent model sets is called a moveme. Then the notion of a model set of being a moveme is relative to a context comprising other model sets. Note that property (i) implies the identifiability property of the model set $M$ (see [6] for example.)

In this paper, we choose our model class $M$ and input $u$ as asymptotically stable linear systems driven by a unit step input with full state output:

$$
\begin{aligned}
\dot{x} & =A x+b \\
y & =x,
\end{aligned}
$$

where $A \in \mathbb{R}^{n \times n}, x=\left(x_{1}, \ldots, x_{n}\right) \in \mathbb{R}^{n}, b \in \mathbb{R}^{n}$, so that $\Theta=(A \mid b) \in E=\mathbb{R}^{n \times(n+1)}$ and $\theta=A \in E^{\prime}=\mathbb{R}^{n \times n}$, with $\Upsilon(A \mid b)=A$. For such a class of models we make the following assumption.

Assumption 2.1. Given $x(t)$ as the output of model (1) we assume that the initial condition $x_{0}$ is such that for any $v \in$ $\mathbb{R}^{n+1}$, we have that $v^{T} \bar{x}(t)=0$, for $t \in\left[t_{1}, t_{2}\right]$, and $t_{2}>t_{1}$, implies $v=0$, where $\bar{x}=\left(x^{T}, 1\right)^{T}$.

This assumption means that the description that model (1) provides for $x(t)$ is minimal in the sense that $x(t)$ cannot also be described by a lower order dynamical system. A direct consequence of such an assumption is given by the following lemma.

Lemma 2.1. Let $x(t)$ and $z(t)$ be generated by two LTI systems

$$
\begin{aligned}
& \dot{x}=A_{1} x+b_{1} \\
& \dot{z}=A_{2} z+b_{2}
\end{aligned}
$$

and let Assumption 2.1 hold. Then $z(t)=x(t)$ for all $t$ if and only if $\left(A_{1} \mid b_{1}\right)=\left(A_{2} \mid b_{2}\right)$.

Proof. $(\Leftarrow)$ If $\left(A_{1} \mid b_{1}\right)=\left(A_{2} \mid b_{2}\right)$ then $z(t)=x(t)$ for all $t$ by uniqueness of solutions.

$(\Rightarrow)$ If $z(t)=x(t)$ for all $t$ then $\dot{z}(t)=\dot{x}(t)$ for all $t$, so that $A_{1} x+b_{1}=A_{2} z+b_{2}$. This implies $\left[\left(A_{1} \mid b_{1}\right)-\left(A_{2} \mid b_{2}\right)\right] \bar{x}(t)=0$ for all $t$, which by Assumption 2.1 (applied to each column) implies $\left(A_{1} \mid b_{1}\right)=\left(A_{2} \mid b_{2}\right)$.

This lemma shows that property (i) of Definition 2.1 is satisfied by our choice of $M$ and $\mathcal{U}$. Property (ii) is verified by choosing for example $\mathcal{C}^{j}, j=1, \ldots, m$, as balls in $\mathbb{R}^{n \times n}$ with centers $A_{c}^{j} \in \mathbb{R}^{n \times n}, j=1, \ldots, m$, and radii $r_{j}$, such that:

$$
\begin{array}{ll}
\mathcal{C}^{j}=B_{r_{j}}\left(A_{c}^{j}\right), & j=1, \ldots, m \\
\mathcal{C}^{j} \bigcap \mathcal{C}^{k}=\{\emptyset\}, & j \neq k
\end{array}
$$

where $m$ is the number of movemes and the matrix norm is the Frobenius norm. In what follows we assume that the sets $\mathcal{C}^{j}$ are described by equation (2). Then we have constructed a set $\mathcal{M}=\left\{M^{1}, \ldots, M^{m}\right\}$ of $m$ movemes where $M^{k}=\left\{M((A \mid b)) \mid A \in \mathcal{C}^{k}\right\}$, for $k \in\{1, \ldots, m\}$ and $M$ is in the form given by equation (1).
Given any signal $x(t)$ we can determine a good representative of such a signal in the class of models (1) by minimizing the cost function (see for example [6]):

$$
(\hat{A} \mid \hat{b})=\underset{(A \mid b)}{\arg \min } \frac{1}{2} \int_{t_{0}}^{T}(\dot{x}-(A \mid b) \bar{x})^{T}(\dot{x}-(A \mid b) \bar{x}) d t
$$

with $\bar{x}=\left(x^{T}, 1\right)^{T}$, which gives the least squares estimate of parameters $(\hat{A} \mid \hat{b})$ so to get the estimate of $x$ in model class (1) as

$$
\dot{\hat{x}}=\hat{A} \hat{x}+\hat{b}, \quad \hat{x}\left(t_{0}\right)=x\left(t_{0}\right) .
$$

In the case in which $x(t)$ has been generated by (1), by virtue of Assumption 2.1 it is easy to check that (3) leads to $(\hat{A} \mid \hat{b})=(A \mid b)$, so that if $A \in \mathcal{C}^{j}$, for some $j \in\{1, \ldots, m\}$ we can classify $x(t)$ as output of moveme $M^{j}$ just by finding $k \in\{1, . ., j, . . m\}$ such that $\hat{A} \in \mathcal{C}^{k}$. This is equivalent by virtue of (2) to finding $k \in\{1, . ., j, . . m\}$ such that $\left\|\hat{A}-A_{c}^{k}\right\| \leq r_{k}$, whose solution is unique since the sets $\mathcal{C}^{k}$ are all not intersecting. Then

$$
\begin{aligned}
\arg _{k \in\{1, ., j, . ., m\}}\left\{\left\|\hat{A}-A_{c}^{k}\right\| \leq r_{k}\right\} & \\
& =\arg _{k \in\{1, ., j, . ., m\}}\left\{\left\|A-A_{c}^{k}\right\| \leq r_{k}\right\}=j
\end{aligned}
$$

In this paper we take a relative straightforward approach to estimation. More sophisticated methods exist for the estimation of parameters in presence of noise. We leave this to future work.

The following section addresses the same classification problem in a more general situation in which $x(t)$ has been generated by a perturbed version of system (1).

\subsection{Classification Problem}

Let the signal $x(t)$ be generated by the perturbed version of system (1):

$$
\begin{aligned}
& \dot{x}=A x+b+d(t) \\
& y=x
\end{aligned}
$$

with $A \in \mathcal{C}^{j}$, for some $j \in\{1, \ldots, m\}$ and $d(t)$ is a bounded realization of white noise. Under what conditions on $A$ and $d(t)$ can we still classify $x(t)$ as output of moveme $M^{j}$ ? Since $A \in \mathcal{C}^{j}$, there exists $\delta<r_{j}$ such that $A=A_{c}^{j}+\delta U$ with $U$ a unit norm matrix and $A_{c}^{j}$ center of $\mathcal{C}^{j}$. Then system (4) becomes

$$
\begin{aligned}
& \dot{x}=\left(A_{c}^{j}+\delta U\right) x+b+d(t) \\
& y=x .
\end{aligned}
$$

Then the problem of classifying $x(t)$ as output of moveme $M^{j}$ becomes the same as identifying $j$ in system (5) for some conditions on $\delta$ and $d(t)$. In the previous section we showed that if $d(t)=0$ then we can exactly identify $A_{c}^{j}+\delta U$ and then correctly classify $x(t)$. The presence of $d(t)$ induces an estimation error so that $\hat{A}$ will not be equal to $A_{c}^{j}+\delta U$, but it is not necessary to achieve equality for our purpose as the following lemma shows.

Lemma 2.2. Let $x(t), t \in\left[t_{0}, T\right]$ be generated by (5), where $A_{c}^{j}$ is the center of $\mathcal{C}^{j}$ for some $j \in\{1, \ldots, m\}$ as in (2). Let 
$\hat{A}$ be the least squares estimate according to (3). There exist positive constants $\bar{d}$ and $\bar{\delta}$ such that if $\delta \leq \bar{\delta}$ and $\|d(t)\| \leq \bar{d}$, then

$$
\arg _{k \in\{1, \ldots j, \ldots m\}}\left\{\left\|\hat{A}-A_{c}^{k}\right\| \leq r_{k}\right\}=j
$$

Proof. By equation (3) we have

$$
(\hat{A} \mid \hat{b})=\left(\int_{t_{0}}^{T} \dot{x}(t) \bar{x}(t)^{T} d t\right)\left(\int_{t_{0}}^{T} \bar{x}(t) \bar{x}(t)^{T} d t\right)^{-1}
$$

where we can invert $\left(\int_{t_{0}}^{T} \bar{x}(t) \bar{x}(t)^{T}\right)$ if either $d(t)=0$ by Assumption 2.1, or $d(t) \neq 0$ by the fact that $d(t)$ is realization of white noise that is uncorrelated in time. Using equation (5), this expression becomes

$$
\begin{aligned}
(\hat{A} \mid \hat{b}) & =\left(A_{c}^{j}+\delta U \mid b\right) \\
& +\left(\int_{t_{0}}^{T} d(t) \bar{x}(t)^{T} d t\right)\left(\int_{t_{0}}^{T} \bar{x}(t) \bar{x}(t)^{T} d t\right)^{-1}
\end{aligned}
$$

which leads with some algebra to

$$
\left\|\hat{A}-A_{c}^{j}\right\| \leq\left\|(\hat{A} \mid \hat{b})-\left(A_{c}^{j} \mid b\right)\right\| \leq \bar{\delta}+\bar{d} c
$$

where $\bar{\delta}$ and $\bar{d}$ are upper bounds on $\delta$ and $d(t)$, and $c$ is a suitable positive constant which exists since $x(t)$ is bounded by the stability properties of the dynamics. Then in order for $\left\|\hat{A}-A_{c}^{k}\right\| \leq r_{k}$ to hold for $j=k$ we require

$$
\left\|\hat{A}-A_{c}^{j}\right\| \leq \bar{\delta}+\bar{d} c \leq r_{j}
$$

which is verified for suitable bounds on $\delta$ and $d(t)$.

\subsection{Well-posedness}

As the previous section highlighted, the basic requirement for solving the classification problem is the one of having non intersecting sets in parameter space characterizing the sets of dynamical models $M^{j}, j=1, \ldots, m$. In practice the sets $\mathcal{C}^{j}$ and $\mathcal{C}^{k}, j \neq k$ may be not defined a priori but are derived from finite number of elements belonging to the sets of signals $\mathcal{S}^{j}$ and $\mathcal{S}^{k}$, whose characteristics make each element of one set different from each element of the other and therefore we can say that they define two classes of signals. When can these two classes of signals define two movemes $M^{j}, M^{k}$ according to Definition 2.1? Let the two classes $\mathcal{S}^{j}$ and $\mathcal{S}^{k}$ be composed by signals $s_{i}^{j}(t)=Y\left(\left.M\left(\Theta_{i}^{j}\right)\right|_{x_{0 i}^{j}, u_{i}^{j}}\right)(t)$, for $s_{i}^{j}(t) \in \mathcal{S}^{j}$, and $s_{i}^{k}(t)=Y\left(\left.M\left(\Theta_{i}^{k}\right)\right|_{x_{0 i}^{k}, u_{i}^{k}}\right)(t)$, for $s_{i}^{k}(t) \in \mathcal{S}^{k}$. Let $\mathcal{F}_{M}$ be an estimation procedure establishing a one to one mapping between the signal $Y\left(\left.M(\Theta)\right|_{x_{0}, u}\right)(t)$ and the couple $(\Theta, u)$ which exists by virtue of (i) of Definition 2.1. Then we have

$$
\begin{aligned}
\left(\Theta_{i}^{k}, u_{i}^{k}\right) & =\mathcal{F}_{M}\left(s_{i}^{k}(t)\right) & s_{i}^{k}(t) \in \mathcal{S}^{k} \\
\left(\Theta_{i}^{j}, u_{i}^{j}\right) & =\mathcal{F}_{M}\left(s_{i}^{j}(t)\right) & s_{i}^{j}(t) \in \mathcal{S}^{j} .
\end{aligned}
$$

Let $f_{s}:(E \times \mathcal{U}) \rightarrow E$ be the selection operator, such that $f_{s}(\Theta, u)=\Theta$, which selects the first element of the couple
$(\Theta, u)$. Then define $f_{M}:=\Upsilon \circ f_{s} \circ \mathcal{F}_{M}$, which associates to each signal $s(t)$ the corresponding parameter $\theta$ lying in $E^{\prime} \subseteq$ $E$. We can then write that $\mathcal{C}^{j}$ is the image of $\mathcal{S}^{j}$ through $f_{M}$ and the same for $\mathcal{C}^{k}$ :

$$
f_{M}\left(\mathcal{S}^{j}\right)=\mathcal{C}^{j}, \quad f_{M}\left(\mathcal{S}^{k}\right)=\mathcal{C}^{k} .
$$

Definition 2.2. Classes of signals $\mathcal{S}^{j}$ and $\mathcal{S}^{k}$ with elements $s_{i}^{j}(t)=Y\left(\left.M\left(\Theta_{i}^{j}\right)\right|_{x_{0 i}^{j}, u_{i}^{j}}\right)(t)$, for $s_{i}^{j}(t) \in \mathcal{S}^{j}$, and $s_{i}^{k}(t)=$ $Y\left(\left.M\left(\Theta_{i}^{k}\right)\right|_{x_{0 i}^{k}, u_{i}^{k}}\right)(t)$, for $s_{i}^{k}(t) \in \mathcal{S}^{k}$, such that the corresponding sets $\mathcal{C}^{j}$ and $\mathcal{C}^{k}$ given in (7) are non intersecting, that is $\mathcal{C}^{j} \cap \mathcal{C}^{k}=\{\emptyset\}$, are said to be well-posed classes according to model $M$.

From this definition it follows immediately that well-posed classes of signals define movemes according to Definition 2.1. In practice we have access to a finite set of signals, $S^{j}=\left\{s_{1}^{j}(t), \ldots, s_{n_{j}}^{j}(t)\right\}$ and $S^{k}=\left\{s_{1}^{k}(t), \ldots, s_{n_{k}}^{k}(t)\right\}$, which belong to the two classes $\mathcal{S}^{j}$ and $\mathcal{S}^{k}$, with $s_{i}^{j}(t)=$ $Y\left(\left.M\left(\Theta_{i}^{j}\right)\right|_{x_{0 i}^{j}, u_{i}^{j}}\right)(t)$ for $i \in\left\{1, \ldots, n_{j}\right\}$ and $s_{i}^{k}(t)=$ $Y\left(\left.M\left(\Theta_{i}^{k}\right)\right|_{x_{0}^{k}, u_{i}^{k}}\right)(t)$ for $i \in\left\{1, \ldots, n_{k}\right\}$. Let $\hat{\mathcal{C}}^{j}$ and $\hat{\mathcal{C}}^{k}$ be the images, through $f_{M}$, of the sets $S^{j}$ and $S^{k}$ respectively. By construction we have $\hat{\mathcal{C}}^{k} \subset \mathcal{C}^{k}$ and $\hat{\mathcal{C}}^{j} \subset \mathcal{C}^{j}$, so that potentially we can have trivial intersection between $\hat{\mathcal{C}}^{j}$ and $\hat{\mathcal{C}}^{k}$, and a no-empty intersection between the sets $\mathcal{C}^{k}$ and $\mathcal{C}^{j}$. As shown in Figure 1, this creates a problem since if we check Definition 2.2 with $\hat{\mathcal{C}}^{j}$ and $\hat{\mathcal{C}}^{k}$, which are the only ones to which we have access, the classes of signals $\mathcal{S}^{j}$ and $\mathcal{S}^{k}$ turn out to be wellposed. The issue comes from the fact that we will use the light

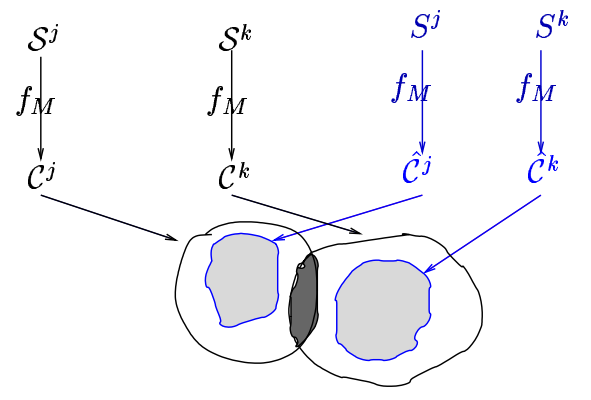

Figure 1: Relation between sets $\hat{\mathcal{C}}^{j}$ and $\hat{\mathcal{C}}^{k}$ and $\mathcal{C}^{j}$ and $\mathcal{C}^{k}$.

sets $\left(\hat{\mathcal{C}}^{j}\right.$ and $\left.\hat{\mathcal{C}}^{k}\right)$ for solving the classification problem ignoring the existence of the dark region that is generating signals with undecidable class. Then, one needs to check if $\mathcal{S}^{k}$ and $\mathcal{S}^{j}$ are well-posed. The following lemma gives a possible way to check for well-posedness without knowing the sets $\mathcal{C}^{j}$ and $\mathcal{C}^{k}$.

Lemma 2.3. Let $y(t)=Y\left(\left.M(\Theta)\right|_{u, x_{0}}\right)(t)$ denote the output of model $M$ for a choice of $\Theta, u$ and $x_{0}$. Assume to fix $u, x_{0}$ and $\left.\Theta\right|_{E-E^{\prime}}$, so that $y(t)=Y(M(\theta))(t)$, and let the classes $\mathcal{S}^{j}$ and $\mathcal{S}^{k}$ be defined as

$$
\begin{aligned}
\mathcal{S}^{j}=\{y(t) \mid y(t) & =Y(M(\theta))(t) \\
& \text { and } \left.g_{j}(y, \dot{y}, t)=0, h_{j}(y, \dot{y}, t) \leq 0\right\}
\end{aligned}
$$




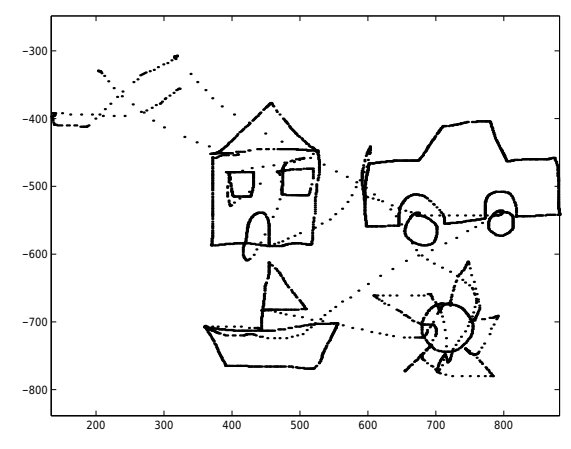

Figure 2: Example of traces in $x y$ plane captured by the capturing system.

$$
\begin{aligned}
\mathcal{S}^{k}=\{y(t) \mid y(t) & =Y(M(\theta))(t) \\
& \text { and } \left.g_{k}(y, \dot{y}, t)=0, h_{k}(y, \dot{y}, t) \leq 0\right\}
\end{aligned}
$$

for some functions $g_{j}, g_{k}, h_{j}$ and $h_{k}$. Then the classes of signals $\mathcal{S}^{j}$ and $\mathcal{S}^{k}$ are well-posed if and only if the system

$$
\begin{array}{r}
y(t)=Y(M(\theta))(t) \\
g_{j}(y, \dot{y}, t)=0 \\
h_{j}(y, \dot{y}, t) \leq 0 \\
g_{k}(y, \dot{y}, t)=0 \\
h_{k}(y, \dot{y}, t) \leq 0
\end{array}
$$

is infeasible.

Proof. $(\Rightarrow)$. Let us show that well-posed classes $\mathcal{S}^{j}$ and $\mathcal{S}^{k}$ imply infeasibility of (8). According to Definition 2.2 this is equivalent to showing that non-intersecting sets $\mathcal{C}^{j}$ and $\mathcal{C}^{k}$ (defined in equation (7)) imply infeasibility of the system of equations (8). Let again $\mathcal{F}_{M}$ be the one to one mapping between the signal $Y\left(\left.M(\Theta)\right|_{x_{0}, u}\right)(t)$ and the couple $(\Theta, u)$. Since input $u$, initial condition $x_{0}$ and $\left.\Theta\right|_{E-E^{\prime}}$ have been fixed, $\mathcal{F}_{M}$ becomes one to one correspondence between $y(t)=Y(M(\theta))(t)$ and $\theta$. Then we can redefine the sets $\mathcal{C}^{j}$ and $\mathcal{C}^{k}$ as

$$
\mathcal{C}^{j}=\left\{\theta \mid \theta=\mathcal{F}_{M}(y(t)), \text { and } y(t) \in \mathcal{S}^{j}\right\}
$$

and

$$
\mathcal{C}^{k}=\left\{\theta \mid \theta=\mathcal{F}_{M}(y(t)), \text { and } y(t) \in \mathcal{S}^{k}\right\} .
$$

If (8) is feasible then there exist $y(t)$ such that $y(t) \in$ $\mathcal{S}^{j}$ and $y(t) \in \mathcal{S}^{k}$ and also there exist $\theta^{*}: y(t)=M\left(\theta^{*}\right)(t)$, so that by (9) and (10) $\theta^{*} \in \mathcal{C}^{j}$ and $\theta^{*} \in \mathcal{C}^{k}$, which in turn implies $\mathcal{C}^{k} \cap \mathcal{C}^{j} \neq\{\emptyset\}$. Then trivial intersection of sets $\mathcal{C}^{j}$ and $\mathcal{C}^{k}$ defined in (9) and (10) implies infeasibility of (8). $(\Leftarrow)$. Let us show that if classes $\mathcal{S}^{j}$ and $\mathcal{S}^{k}$ are not wellposed, then system (8) is feasible. By Definition 2.2, this is equivalent to show that if $\mathcal{C}^{k} \cap \mathcal{C}^{j} \neq\{\emptyset\}$ then system (8) is feasible. $\mathcal{C}^{k} \cap \mathcal{C}^{j} \neq\{\emptyset\}$ implies that there exist $\theta^{*} \in \mathcal{C}^{j}$ and $\theta^{*} \in \mathcal{C}^{k}$ which from (9) and (10) implies that there exist a signal $y^{*}(t)$ such that $\theta^{*}=\mathcal{F}_{M}\left(y^{*}(t)\right), y^{*}(t) \in \mathcal{S}^{j}$ and $\theta^{*}=\mathcal{F}_{M}\left(y^{*}(t)\right), y^{*}(t) \in \mathcal{S}^{k}$, which means that the signal $y^{*}(t)$ is both in $\mathcal{S}^{j}$ and $\mathcal{S}^{k}$ which implies that it satisfies (8), then (8) is feasible.

\section{Experiments}

To test our approach, we studied a 2D drawing task in which a set of shapes were drawn by five different subjects using a computer mouse.

\subsection{Experimental setup}

Our subjects drew using the XPaint program on a PC running Red Hat Linux 7.2 with a screen measuring $1600 \times 1200$ pixels and a working window of $700 \times 500$ pixels. The user left the trace of the trajectory in the working window only when the left mouse button was pressed. For acquiring $x$ and $y$ time traces we implemented a $\mathrm{C}$ routine which was activated in the background at the beginning of each experimental session and sampled the $(x, y)$ position of the pointer everywhere on the screen at the rate of $100 \mathrm{~Hz}$ and a spatial resolution of one pixel. The time interval between one sample and the following one turned out to be mostly constant except for slight variations every once in a while due to higher priority of other processes. In order to have constant sampling time the data was processed through an algorithm that linearly interpolates data in the regions in which the time interval is not exactly $10 \mathrm{~ms}$. Pixelization of the coordinates does not heavily affect the data since the trajectories under study are usually more than 50 pixels long.

We defined 4 different drawings by means of prototypes: car, sun, ship, and house. Each of the 5 subjects was shown the prototypes and was asked to reproduce them on a $700 \times 500$ pixel canvas; the dimensions of each drawing could be chosen arbitrarily according to the ones with which the user was more comfortable, the only specification was to reproduce the prototypes with as high fidelity as possible in a reasonable amount of time. Some of the data captured is shown in Figure 2. Each subject drew 10-20 examples for each shape. In order to accomplish each drawing task the user had to perform a sequence

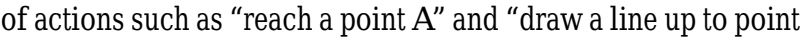
$B$ ". These actions are the ones that we will consider as candidates for being elementary motions and then defining a pair of movemes. Thus we check if reach and draw actions define a well posed pair of movemes according to Definition 2.3.

\subsection{Classification}

We start from the hypothesis that "draws", which are straight lines traced with a specific intention (like drawing a side of the house), and "reaches", which happen with the intention of shifting fast the equilibrium position, define a well-posed pair of movemes. We segmented out by hand a set of straight draws from houses and cars drawn by 2 of the subjects. Reach examples were obtained from a special experiment session in which the users had to point and click at random buttons appearing on a $700 \times 500$ pixels window during a simple video game implemented in MATLAB 6.0.

We considered several discrete time dynamical models for representing the reach and draw signals, starting from a first order, 

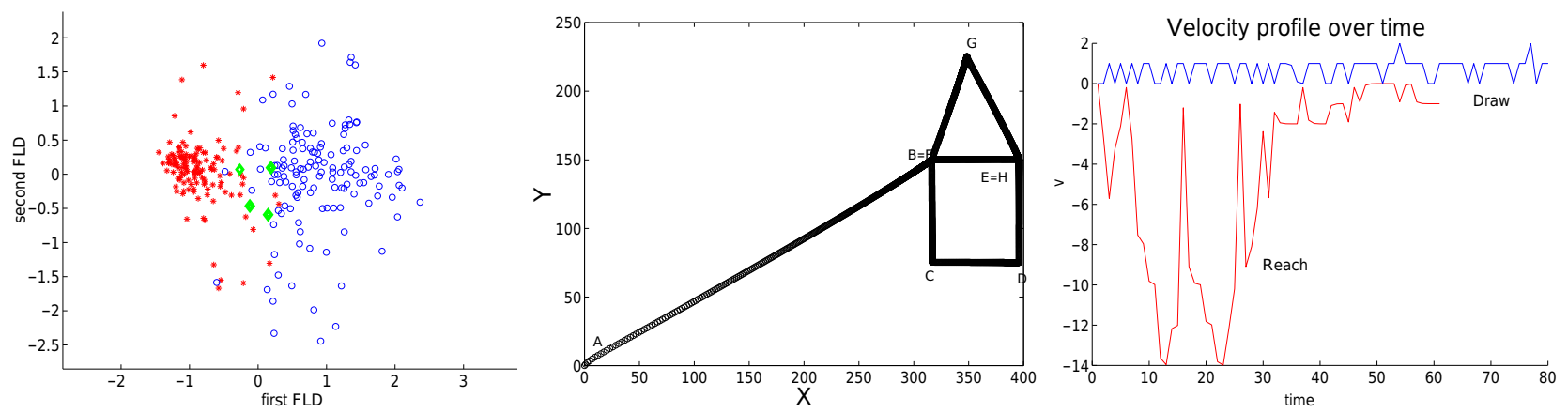

Figure 3: Parameter estimates for reach (stars) and draw (circles) examples projected on the first two Fisher linear discriminants (left). Diamonds represent some of the parameters corresponding to straight $(x, y)$ trajectories. House generated by some of the diamonds of the left picture (center). Typical reach and draw velocity profiles (right).

decoupled model for $x$ and $y$ motion,

$$
\begin{aligned}
& \dot{x}_{k+1}=a_{1 x} x_{k}+b_{x} \\
& \dot{y}_{k+1}=a_{1 y} y_{k}+b_{y},
\end{aligned}
$$

and proceeding to a second order coupled model,

$$
X_{k+1}=\left(\begin{array}{cccc}
0 & 1 & 0 & 0 \\
a_{1 x} & a_{2 x} & a_{3 x} & a_{4 x} \\
0 & 0 & 0 & 1 \\
a_{3 y} & a_{4 y} & a_{1 y} & a_{2 y}
\end{array}\right) X_{k}+\left(\begin{array}{c}
0 \\
b_{x} \\
0 \\
b_{y}
\end{array}\right)
$$

where $X_{k}=\left(x_{k-1}, x_{k}, y_{k-1}, y_{k}\right)$. The reach dynamical parameters were estimated from 140 examples of reach trajectories obtained from the video game implemented in MATLAB, and the draw dynamical parameters were estimated from 140 examples of draw trajectories segmented out from cars and houses of 2 of the subjects. The dynamical parameters were estimated for each one of the dynamical models proposed (first order for $x$ and $y$, decoupled; first order for $x$ and $y$, coupled; second order for $x$ and $y$, decoupled; second order for $x$ and $y$, coupled).

By proceeding with standard pattern recognition techniques (see [1] for example), we trained a Gaussian classifier for the parameters derived from the 140 examples per class (training set) for each one of the model classes proposed, and obtained the best results for the second order for $x$ and $y$, decoupled, dynamical model (obtained by letting $a_{3 x}=0, a_{4 x}=0, a_{3 y}=0$, $a_{4 y}=0$ in system (11)):

$$
X_{k+1}=\left(\begin{array}{cccc}
0 & 1 & 0 & 0 \\
a_{1 x} & a_{2 x} & 0 & 0 \\
0 & 0 & 0 & 1 \\
0 & 0 & a_{1 y} & a_{2 y}
\end{array}\right) X_{k}+\left(\begin{array}{c}
0 \\
b_{x} \\
0 \\
b_{y}
\end{array}\right)
$$

For such a model we obtained $3.2 \%$ training error, and we tested the generalization properties of the resulting classifier on a test set of 323 additional reach examples (obtained from the MATLAB videogame) and 118 additional draw examples obtained from the drawings of other two subjects (different from the ones used for the training set) and obtained $3.63 \%$ test error. Figure 3 (left) represents the projection of the parameters belonging to the training set (living in $\mathbb{R}^{4}$ ) on the first two Fisher linear discriminants [1] and typical velocity profiles for the draw and reach trajectories. We let $\hat{\mathcal{C}}^{R}$ and $\hat{\mathcal{C}}^{D}$ denote the reach and draw clusters, respectively, according to the notation used in Section 2.3.

\subsection{Well-posedness}

By looking at the sets $\hat{\mathcal{C}}^{R}$ and $\hat{\mathcal{C}}^{D}$ of Figure 3 (left) one notice that we have a quite good separation. Anyway, since these two sets are just estimates of the real ones $\mathcal{C}^{R}$ and $\mathcal{C}^{D}$, we have to check that situation depicted in Figure 1 does not happen. To check this, we find candidate constraints which can describe reach and draw trajectories, so that we may apply Lemma 2.3. Reach trajectories are asymptotically stable with bell-shaped velocity profiles. Draw trajectories are characterized by asymptotic stability properties and by straight lines in $x y$ plane. These characteristics are not incompatible with each others. The diamonds shown in Figure 3(left) are parameters corresponding to straight lines in $x y$ plane ( $a_{1 x}=a_{1 y}$ and $a_{2 x}=a_{2 y}$ ), and we see clearly that they can be both reach and draw parameters. We have a situation analogous to the one reported in Figure 1, where the light sets are $\hat{\mathcal{C}}^{D}$ and $\hat{\mathcal{C}}^{R}$ and the dark set is made up by elements like the diamonds in Figure 3(left). Thus there exist parameters that generate trajectories satisfying both draw and reach constraints whose class is undecidable. As an extreme example of this, we show in Figure 3 (center) the shape of a house that has been artificially generated by parameters lying in the region in between the clusters of Figure 3 (left), which the classifier classifies as reaches.

This happens because the dynamical parameters associated to draw trajectories are task dependent, and in some cases they correspond to straight trajectories with bell shaped velocity profiles (as it happens in the rays of the sun). We show these differences in Figure 4, where we report the draw parameters when a user draws straight lines between two points (as it hap- 

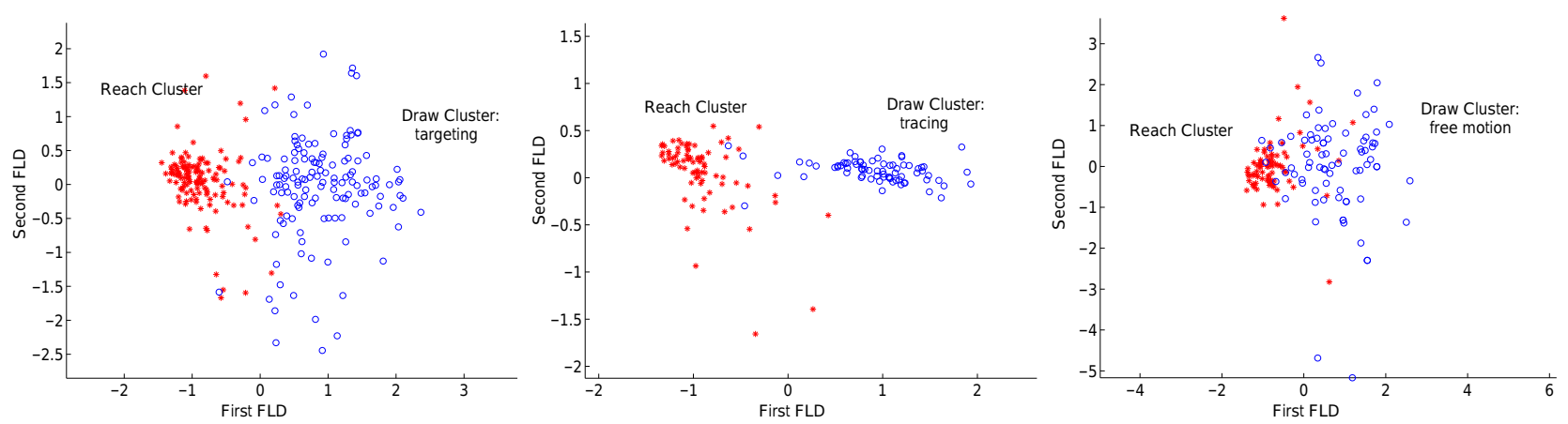

Figure 4: Parameter estimates for reach and draw targeting, draw tracing and free motion draw.

pens in the draws of the house, ship, car), or a line trying to trace an already existing line, or just a line with no constraints (as it happens in the rays of the suns). We decide therefore to use three classes instead of one for the draw: we call them targeting, tracing, and free motion respectively.

From Figure 4 we see that there is an evident overlapping of the parameter sets of the reach class and free motion class. Therefore we exclude from the panorama the free motion class, and show that the draw class, seen as union of the tracing and targeting motions, can be described in terms of constraints $g_{D}$, $h_{D}, g_{R}, h_{R}$ as introduced in Lemma 2.3, such that the system of equations (8) is infeasible. Driven by the characteristics of the velocity profiles of the targeting and tracing draw (almost constant velocity) and reach (bell shaped velocity profile), as shown in Figure 3 (right), we define the following constraints. The reach trajectories should be characterized by a certain acceleration peak, and the draw trajectories should be characterized by a small velocity. We then rewrite these constraints in the form of Lemma 2.3 as $\dot{x}-a \dot{y}=g_{D}(\dot{x}, \dot{y})=0$ and $b-\|(\ddot{x}, \ddot{y})\|=h_{D}(\ddot{x}, \ddot{y}) \geq 0$ for the draw motions, and $\|(\ddot{x}, \ddot{y})\|-c=h_{R}(\ddot{x}, \ddot{y}) \geq 0$ for the reach motion, so that the system of inequalities (8) becomes infeasible for suitable $b$ and $c$. Then if we assume that the constraints above define fair specifications for reach and draw trajectories for the values of $b$ and $c$ that make system (8) infeasible, then the reach and draw classes of signals are well-posed according to Lemma 2.3. Moreover the $\hat{\mathcal{C}}^{R}$ and $\hat{\mathcal{C}}^{D}$ clusters of Figure 3 (left) well represent reach and draw actions, which thus define a pair of movemes $M^{R}$ and $M^{D}$.

\section{Conclusions}

We have provided the definition of well-posed sets of signals. We provided an operative way to check if sets of actions are well-posed according to a dynamical model class $M$, and thus they can generate movemes. We have tested our ideas on human drawing data and discovered two sets of actions (reach and draw), which can define movemes and one set of actions (free motion) that is not well-posed according to the already formed alphabet of reach and draw movemes.

\section{Acknowledgements}

This project has been funded in part by the NSF Engineering Research Center for Neuromorphic Systems Engineering (CNSE) at Caltech (NSF9402726). The authors would like to acknowledge all the people who participated to the experiments.

\section{References}

[1] C.M. Bishop. Neural Networks for Pattern Recognition. Clarendon, Oxford, 1995.

[2] C. Bregler and J. Malik. Learning and recognizing human dynamics in video sequences. In Proc. IEEE Conference on Computer Vision and Pattern Recognition, pages 568674, Puerto Rico, 1997.

[3] D. DelVecchio, R. M. Murray, and P. Perona. Segmentation of human motion into dynamic based primitives with application to drawing tasks. In Submitted to the 2003 American Control Conference, Denver, Colorado, June 4-6.

[4] D. DelVecchio, R. M. Murray, and P. Perona. Primitives for human motion: a dynamical approach. In Proceedings of the 2002 IFAC 15th World Congress, Barcelona, Spain, 2002.

[5] L. Goncalves, E. Di Bernardo, and P. Perona. Reach out and touch space (motion learning). In Proc. of the Third International Conference on Automatic Face and Gesture Recognition, pages 234-239, Nara, Japan, April 14-16 1998.

[6] L. Ljung. System Identification. Prentice Hall, New Jersey, 1999.

[7] T. Söderström and P. Stoica. System Identification. Prentice Hall. Hemel Hempstead, 1989.

[8] V. Vapnik. The Nature of Statistical Learning Theory. Springer Verlag, 1995. 